Claremont Colleges

Scholarship@ Claremont

Scripps Faculty Publications and Research

Scripps Faculty Scholarship

$1-1-2000$

\title{
Age Differences in Behavior and PET Activation Reveal Differences in Interference Resolution in Verbal Working Memory
}

\author{
Alan Hartley \\ Scripps College \\ John Jonides \\ University of Michigan \\ Christina Marshuetz \\ University of Michigan \\ Edward E. Smith \\ University of Michigan \\ Patricia A. Reuter-Lorenz \\ University of Michigan \\ See next page for additional authors
}

\section{Recommended Citation}

Jonides, J., Smith, E. E., Reuter-Lorenz, P., Koeppe, R. A., \& Hartley, A. (2000). Age differences in behavior and PET activation reveal differences in interference resolution in verbal working memory. Journal of Cognitive Neuroscience, 12, 188-196. doi: 10.1162/ 089892900561823 
Authors

Alan Hartley, John Jonides, Christina Marshuetz, Edward E. Smith, Patricia A. Reuter-Lorenz, and Robert A. Koeppe 


\title{
Age Differences in Behavior and PET Activation Reveal Differences in Interference Resolution in Verbal Working Memory
}

\author{
John Jonides, Christina Marshuetz, Edward E. Smith, \\ Patricia A. Reuter-Lorenz, and Robert A. Koeppe \\ University of Michigan
}

\author{
Alan Hartley \\ Scripps College
}

\begin{abstract}
Older adults were tested on a verbal working memory task that used the item-recognition paradigm. On some trials of this task, response-conflict was created by presenting test-items that were familiar but were not members of a current set of items stored in memory. These items required a negative response, but their familiarity biased subjects toward a positive response. Younger subjects show an interference effect on such trials, and this interference is accompanied by activation of a region of left lateral prefrontal cortex. However, there has
\end{abstract}

\section{INTRODUCTION}

The human frontal lobes play a central role in mediating executive processes of cognition, processes such as resolving interference between alternative responses and managing multiple tasks simultaneously (for example, Milner, Petrides, \& Smith, 1985; Roberts, Hager, \& Hernon, 1994; West, 1996; Smith, Jonides, Marshuetz, \& Koeppe, 1998). The frontal lobes are also especially susceptible to atrophy and to the adverse physiological effects of normal aging (Huttenlocher, 1979; Warren, Butler, Katholi, \& Halsey, 1985; Rinne, 1987; Fuster, 1989; Haug \& Eggers, 1991; Cowell et al., 1994; Raz et al., 1997; Raz, Gunning, Head, Dupuis, \& Acker, 1998; Raz, in press). These neurophysiological changes should presumably lead to deficits in the executive processes that depend on frontal structures. Indeed, Moscovitch and Winocur (1995) have reviewed the effects of frontal damage on a number of such executive processes.

One class of executive process resolves conflict between competing processes or responses in tasks that include color naming (Perret, 1974), attending to selected regions of text (Connelly, Hasher, \& Zacks, 1991), withholding a response to a presented stimulus (Drewe, 1975), making a saccade away from the loca- been no evidence that the activation in this region is causally related to the interference that the subjects exhibit. In the present study, we demonstrate that older adults show more behavioral interference than younger subjects on this task, and they also show no reliable activation at the same lateral prefrontal site. This leads to the conclusion that this prefrontal site is functionally involved in mediating resolution among conflicting responses or among conflicting representations in working memory.

tion of a presented target (Guitton, Buchtel, \& Douglas, 1985), and others (Carter et al., 1998). What ties such cases together is that in all these tasks, a response is prepotent, but this response must be withheld in favor of another response that is correct on a given trial. Thus, these cases all involve resolving conflict between one process and another by selecting one process and inhibiting the other. In all these cases, the frontal lobes have been implicated as critical to conflict resolution. This is shown by neuroimaging studies in which there is activation in frontal lobes during such tasks (e.g., Carter et al., 1998), and by studies of patients who have damage to regions of the frontal lobes and show deficits in the performance of these tasks (e.g., Guitton et al., 1985).

We have previously tested a verbal working memory task that involves this same sort of conflict-resolution (Jonides, Smith, Marshuetz, \& Koeppe, 1998). The working memory task we used was item-recognition: A target-set of four letters was presented to subjects followed by a retention interval of three sec and then a single probe-letter. Subjects gave one response if the probe was a member of the target-set and another if it was not. We constructed trials in the critical condition of this experiment such that a probe on a current trial 
that required a negative response was often a member of the previous trial's target-set. Thus, this probe-item had a high familiarity that would predispose subjects to respond positively, even though it required a negative response on the current trial. Young adults (ages 19-30) showed interference on such trials compared to control trials, and they also showed activation in a region of left lateral prefrontal cortex in Brodmann's area (BA 45) (Jonides et al., 1998; D'Esposito, Postle, Jonides, Smith, \& Lease, in press). In that this activation was accompanied by poorer performance, we were led to the conclusion that it was functionally involved in mediating performance on these interfering trials. However, this conclusion must be considered tentative in that it is based only on a correlation between behavioral performance and brain activation.

To establish that activation in area 45 mediates the behavioral interference effect, we compared this activation in two groups of individuals who should differ in their susceptibility to interference in this task. We reasoned that older adults compared to younger adults would show greater interference on this task because of age-related decline in frontal function (for example, Raz, in press; Warren et al., 1985), and because of increased problems with inhibitory processes (for example, Connelly et al., 1991). We reasoned that increased interference in older adults may be accompanied by a change in activation in the critical left lateral site that we had found activated in the younger subjects. If the activation decreased in magnitude with decreased ability to resolve interference, this would implicate the site as part of a mechanism that intervened when interference was present in processing. If the activation increased in magnitude with decreased interference resolution, this might imply that older adults had to engage this mechanism even more than younger adults, but unsuccessfully so.

The task we used is illustrated in Figure 1. There were two experimental conditions in which subjects had to remember a target-set of four letters for $3 \mathrm{sec}$, after which a probe letter was presented. The probe matched one of the target letters on half the trials (positive probes); on the other half it did not match any target (negative probes).

In one of the experimental conditions (the HighRecency condition), half of the negative-probes did match a target from the immediately previous trial even though these probes demanded a "no" response on the current trial (call these "recent negative probes"). Thus, trials with recent negative probes present subjects with a conflict between the high familiarity of the probe (suggesting a positive response) and its lack of membership in the target set of the present trial (demanding a negative response). The probe on the remaining negative trials neither matched a target from the present trial nor one from either of the two previous trials (call these "non-recent negative probes").

We found that recent negative probes resulted in longer response times than non-recent negative probes, which replicates previous results (Monsell, 1978). Furthermore, we documented that the HighRecency condition yielded brain activation in BA 45 compared to a Low-Recency condition in which all negative probes were of the non-recent variety (Jonides et al., 1998). This result has since been confirmed using functional magnetic resonance imaging (fMRI) and a randomized-trials design by D'Esposito

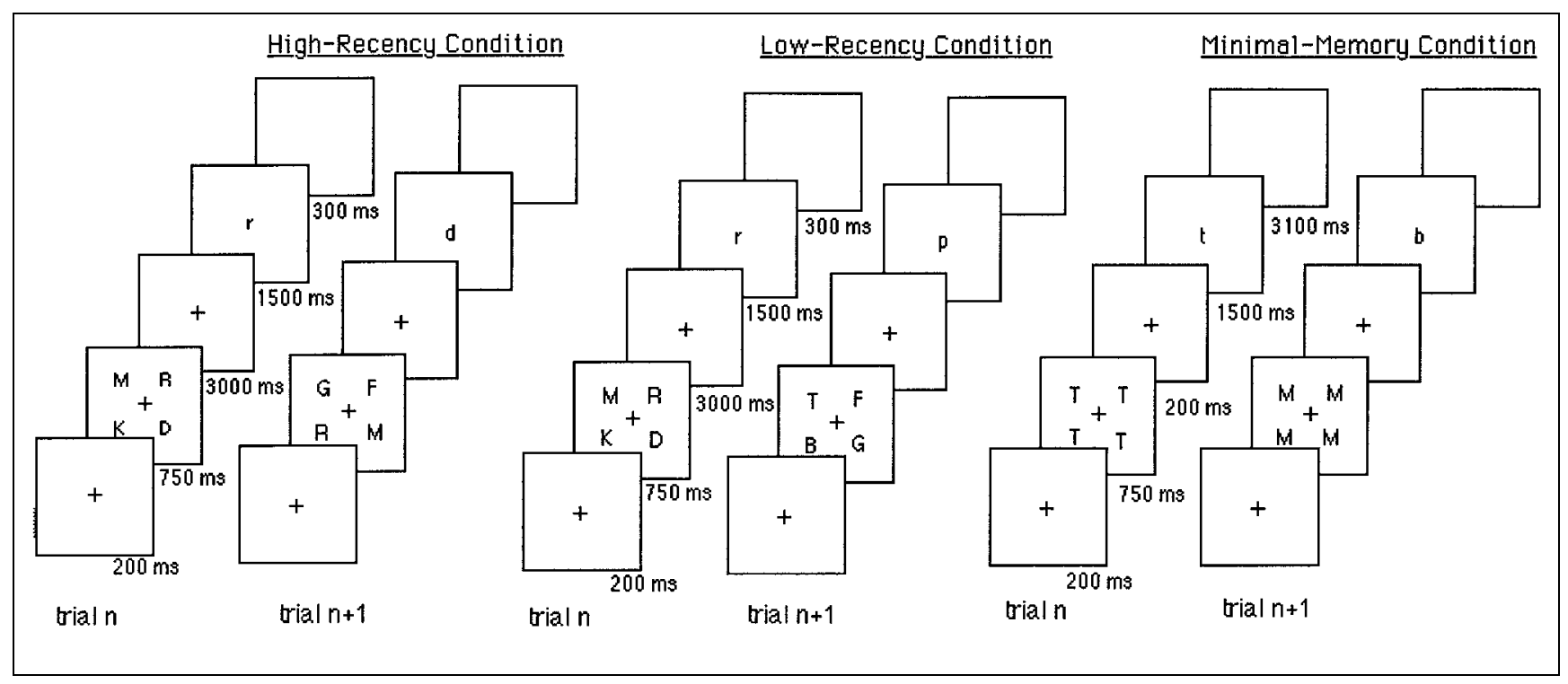

Figure 1. Schematics of the three conditions used in this experiment: High-Recency, Low-Recency, and Minimal-Memory. See the text for important features of these conditions. Shown are example events in each condition with durations (in msec) for each event. 
et al. (in press), so it is not an artifact of the blocked design that was necessitated by our earlier positron emission tomography (PET) experiment. This prefrontal site, then, appears to be implicated in processes that resolve conflict between interfering memory representations.

Age may be expected to adversely affect interference resolution in that it has been suggested by various researchers that older adults suffer a deficit in the inhibitory processing that may be crucially involved in this process (e.g., Hasher \& Zacks, 1988; Tipper, 1991; West, 1996). Although the precise nature of this inhibitory deficit has not been well-characterized, there have been various demonstrations of failures by older adults to inhibit the entry of irrelevant information into working memory, to inhibit prepotent responses, or to inhibit contextually inappropriate mental processes more generally (e.g., Connelly et al., 1991). In that inhibitory processes may be an important component of the processing required to resolve a conflict between alternative representations, these demonstrations of inhibitory failures in older adults led us to the predictions that: (a) older adults would suffer an even greater difference in performance between recent and nonrecent negative probes in our task than younger adults; and (b) this failure to inhibit would be accompanied by a lower level of brain activation in the lateral prefrontal site we have identified with inhibitory processing in younger adults if this site mediates interference-resolution processes.

We evaluated these predictions by testing a group of 12 older adults (ages 61-72) using PET measurements in precisely the same experiment in which we had previously tested younger adults. The High- and Low-Recency conditions, described above, constituted the two experimental conditions. In addition, we included a Minimal-Memory control condition in which a single letter was the target, and the retention interval between the target and probe letters was only $200 \mathrm{msec}$ (see Figure 1). The Minimal-Memory control condition required the same task as the experimental conditions, but with a substantially reduced memory load and retention interval.

\section{RESULTS AND DISCUSSION}

The critical feature of behavioral performance for the older adults is their response to the recent negative probes in the High-Recency condition (Table 1). These trials yielded a mean response time of 926 msec and a mean accuracy of $97 \% .{ }^{1}$ There are two statistical comparisons that demonstrate an interference effect on these trials, both accomplished via $t$ tests. One comparison reveals that response times are slower than on trials with non-recent negative probes in the High-Recency condition (which had a mean response time of $869 \mathrm{msec}$ and a mean accuracy of $98 \%)(p=.016)$. The second comparison shows that the recent negative probes of the High-Recency condition resulted in slower responses than the negative probes of the Low-Recency condition (which had a mean response time of $874 \mathrm{msec}$ and a mean accuracy of 92\%; note, though, that this second comparison may be tainted by a lower accuracy in this condition) $(p=.027)$.

Our previous data with younger adults showed this same pattern of interference. Importantly, however, we compared the size of the interference from our previous study of younger adults with that from the current study of older adults, and we found that the effect for older adults is larger than that for younger adults. In order to effect this comparison, we created a single index of interference that combined the effects shown in the response times and accuracies in that both measures are potential indices of interference. To compute this measure, we used the following procedure, following Salthouse (1992): First, for each of the older and younger subjects, we created a difference score between that person's performance on the trials with recent negative probes (in Table 1, these are labeled "Recent No") and the trials with non-recent negative probes (labeled "Non-Recent No"). This was

Table 1. Latency and Accuracy from PET Session

\begin{tabular}{|c|c|c|c|c|c|}
\hline \multirow[b]{2}{*}{ Condition } & \multirow[b]{2}{*}{ Response Type } & \multicolumn{2}{|c|}{ Young Adults } & \multicolumn{2}{|c|}{ Older Adults } \\
\hline & & $R T(m s e c) \pm S E$ & Accuracy (\%) $\pm S E$ & $R T(m s e c) \pm S E$ & Accuracy (\%) $\pm S E$ \\
\hline \multirow[t]{2}{*}{ Minimal-Memory } & Yes & $529 \pm 23$ & $98 \pm 1.2$ & $557 \pm 42$ & $99 \pm 0.5$ \\
\hline & No & $596 \pm 26$ & $98 \pm 1.9$ & $676 \pm 42$ & $97 \pm 2.2$ \\
\hline \multirow[t]{2}{*}{ Low-Recency } & Yes & $683 \pm 34$ & $99 \pm 0.4$ & $815 \pm 41$ & $95 \pm 2.2$ \\
\hline & No & $741 \pm 30$ & $99 \pm 0.6$ & $874 \pm 30$ & $92 \pm 2.6$ \\
\hline \multirow[t]{4}{*}{ High-Recency } & Non-Recent Yes & $695 \pm 26$ & $99 \pm 0.5$ & $821 \pm 40$ & $98 \pm 0.8$ \\
\hline & Recent Yes & $688 \pm 33$ & $99 \pm 0.5$ & $802 \pm 44$ & $96 \pm 0.9$ \\
\hline & Non-Recent No & $739 \pm 33$ & $98 \pm 0.1$ & $869 \pm 38$ & $98 \pm 0.8$ \\
\hline & Recent No & $772 \pm 33$ & $99 \pm 0.4$ & $926 \pm 34$ & $97 \pm 1.3$ \\
\hline
\end{tabular}


done separately for mean response times and mean accuracies. We then created a pool of all the responsetime scores for both groups of subjects, and calculated the $z$ score value for each subject in that pool. We did the same for the accuracies. We then combined the $z$ scores for response time and accuracy by subtracting the response time $z$ score from the $z$ score for accuracy (subtraction was used because better performance is indicated by higher accuracy but by lower response time, and so subtraction adjusts for this reciprocal relationship). The result was an overall $z$ score for each subject in each age group that was an index of how much interference that subject showed on trials with recent negative probes compared to non-recent negative probes. A comparison of these $z$ scores for the older subjects and the younger subjects revealed a reliable difference between the age groups $(p=.022)$. Table 1 displays the mean response times and accuracies on which this analysis was based. The table shows what the analysis confirms: that adults in general show an interference effect when a probe that previously would have required a positive response requires a negative response on the current trial, and that older adults suffer disproportionally more from this interference effect than younger adults.

Our previous data with younger adults implicated area 45 left lateral prefrontal cortex as critical to this interference effect. To evaluate the activation in this site in the older adults and to compare the activation at this site between older and younger adults, we conducted two region of interest (ROI) analyses. First, we created an ROI by combining the activations of the older and younger adults in the High-Recency condition and subtracting the combined activations of the two age groups in the Low-Recency condition. The resulting subtraction revealed only one single peak voxel of reliable activation in left lateral prefrontal cortex that we designated the peak of the region relevant to interference resolution (coordinates: $x=-51, y=21, z=11$, BA 45). We constructed an ROI around this peak by defining a sphere with diameter equal to seven voxels $(2.25 \mathrm{~mm}$ on a side per voxel; this region contains 180 voxels, with 88 voxels equivalent to $1 \mathrm{ml}$ of volume; thus the ROIs are a bit larger than $2 \mathrm{ml}$ in volume). We then evaluated whether this spherical volume was reliably activated in each of the subject-groups separately by $t$ tests that combined the activations in all the voxels within the ROI for each age group. We found that there was reliable activation at this site in the younger subjects $(p=.01)$, but only a marginally reliable effect in the older subjects $(p=.07)$. Importantly, the difference in activation at this site between the younger and older adults was reliable, as assessed by a between-subjects $t$ test that compared the magnitude of the effect in the older subjects with that in the younger subjects $(p=.045)$.
The analysis just presented, of course, relies on a somewhat arbitrary ROI in that this ROI was specified as a sphere surrounding a peak voxel. The merit of this analysis is that it creates an ROI that is symmetrical around the voxel of greatest activation in the combined data of the young and the old. However, it may not properly reflect the truly activated voxels within this region because of its arbitrary shape. In order to address this possibility, we conducted a second ROI analysis. In this analysis, we again created an ROI by subtracting activations in the Low-Recency condition from those in the High-Recency condition for the younger and older subjects combined. This ROI, however, was not arbitrarily spherical as in the previous analysis. Rather, we created this ROI by beginning with the peak voxel at coordinates $x=-51, y=21, z=11$, and we grew a region by identifying all contiguous voxels that were activated using a criterion of $z$ equal to or greater than 3.0. This produced a volume containing 194 voxels. We then placed this volume on the data of the younger and older subjects separately. For the younger subjects, the activation in this ROI was highly reliable $(p=.002)$. For the older subjects, it was also reliable, although less so $(p=.04)$. Importantly, a comparison of activation in this ROI between older and younger subjects revealed, as in the previous analysis, a significant difference $(p=.044)$. Indeed, removing from this analysis two outlying subjects, one older and one younger, strengthened the significance of the difference $(p=.026)$.

Visually, the difference between older and younger subjects can be appreciated by examining Figure 2, which shows brain activations superimposed on surface-rendered images of left-lateral, superior, and rightlateral views of the brain. For purposes of comparison, we have included in this figure images from the younger subjects of the previous study. The images include; all activations that passed a significance criterion of $p<.05$ without correcting for multiple comparisons, thus, it includes many activations that would not pass a strict criterion for significance. After a correction for multiple comparisons was applied to the data, only the activation in the left-lateral prefrontal cortex in the young subjects was reliable. What is striking about these visual images is that the younger subjects clearly show greater activation in the left lateral region than the older subjects, the finding that is confirmed by our ROI analyses.

However, the figure also suggests that older and younger subjects may differ in activations in other regions in addition to the left lateral prefrontal region. This is not a statistically confirmed suggestion, as our analysis above indicates; however we were concerned that there might, nonetheless, be other regions in which younger subjects also showed some evidence of greater activation than older subjects, in addition to the left-lateral prefrontal region. If this were so, it 
might indicate that there was a distributed circuitry involved in interference resolution even though our direct comparison of younger and older subjects on the subtraction of activation in High vs. Low-Recency conditions did not provide any evidence of reliable activation differences in regions other than left lateral prefrontal cortex. We conducted an additional analysis to examine whether other regions might also differ as a function of age.

To identify additional regions to examine, we could not use the direct subtraction of High-Recency vs. LowRecency conditions because this subtraction reveals reliable activation in just the left lateral prefrontal site, as we describe above. Instead, we examined the activations for High-Recency vs. Low-Recency, a subtraction that should reveal regions involved more generally in verbal working memory. The subtraction was accomplished on the pooled data of the younger and older participants, and it yielded 16 peak voxels with $z$ scores greater than or equal to 4.0 . The $(x, y, z)$ coordinates of these voxels with their associated significances are: $(26,-58,40: 8.72)$, (48, -4, 36: 8.27), (37, 8, 25: 6.83),
$(-3,-60,-18: 6.26),(-10,-62,-18: 6.23),(19,1,43$ : 5.79), (39, -62, -16: 5.74), (-26, -58, -22: 5.68), $(-28,-58,34: 5.59),(30,-37,34: 5.58),(17,-8,25$ : $5.34),(-28,-64,-47: 5.33),(19,-60,-18: 4.35)$, $(-17,17,22: 4.22),(3,-35,-25: 4.12),(6,-82,-29$ : 4.04). (Note that this list does not include a separate site for area 45 in the left hemisphere; as Figure 2 suggests, this site is part of a larger swath of activation that extends superiorly and posteriorly, and it did not appear as a separate site in this analysis because of its close proximity to other sites that did appear in this locale.) We then created spherical ROIs from these peak voxels using the method described above, and we placed these ROIs on the difference images for the High-Recency vs. Low-Recency subtraction separately for the older and younger subjects. Comparing the activations in the two age groups at the sites of these ROIs revealed that none was reliably different using even a loose criterion of $p<.05$ uncorrected for multiple comparisons. To be complete, we then repeated this analysis using the subtraction of the Minimal Memory condition from the average of the High- and

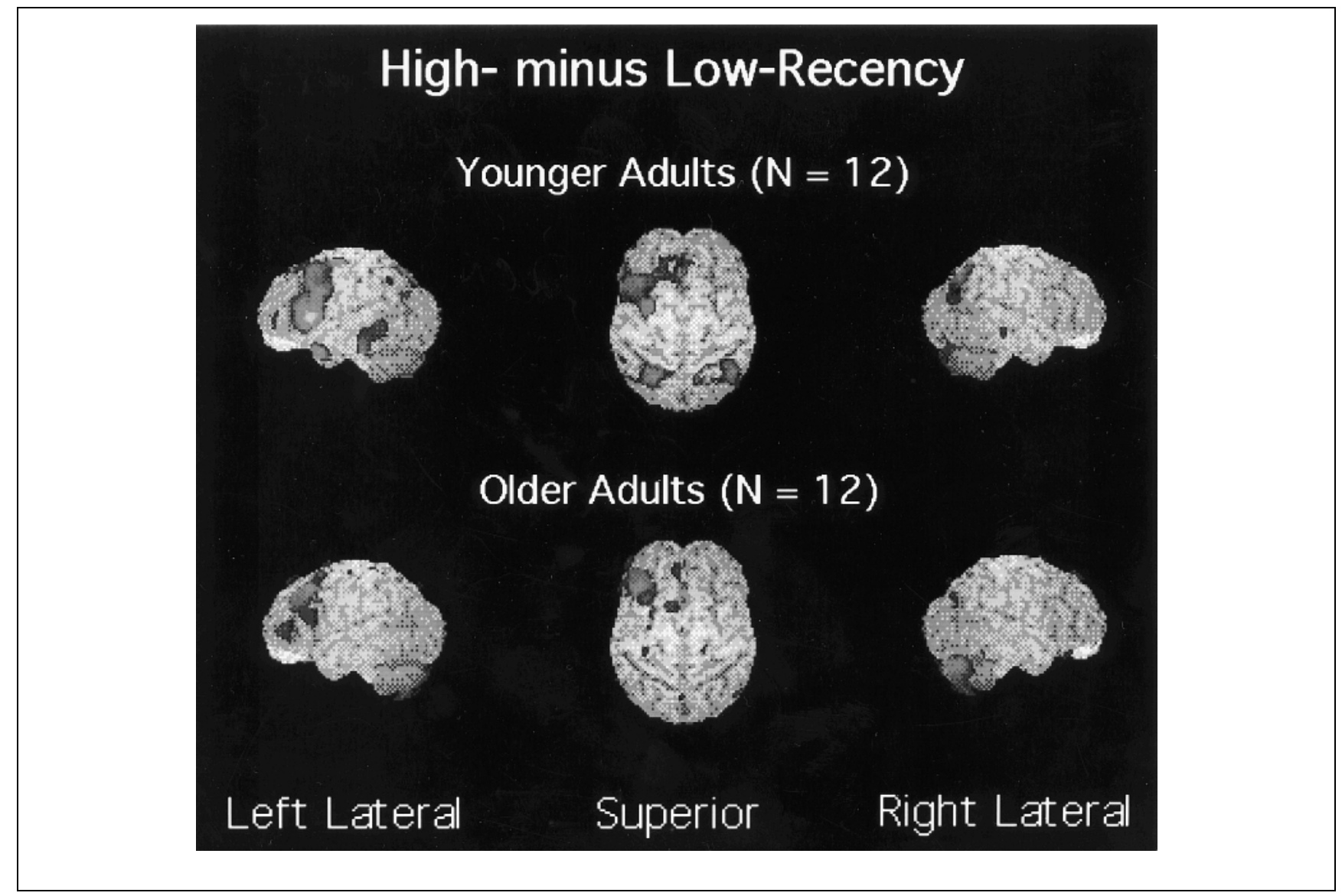

Figure 2. Surface-rendered images of activations for older adults from the present experiment; for purposes of comparison, similar images are also shown for a previous study with younger adults (Jonides et al., 1998). The three views shown are left lateral, superior, and right lateral. Superimposed on a representative MRI of the brain (not of a subject in this experiment) are activations in a rainbow color scale showing $t$ statistics of activations at or within $15 \mathrm{~mm}$ of the surface of the brain. The color scale ranges from yellow at its highest to blue at its lowest. Shown are all activations that passed a criterion of $p<.05$ uncorrected for multiple comparisons. All activations are from a subtraction of the Low-Recency from the High-Recency condition. 
Low-Recency conditions. This analysis yielded 22 peak voxels of activation in the combined data of the younger and older subjects. Of these, only one showed that younger subjects had higher activation than older subjects at $p=.03$, and even this difference would not have survived a correction for multiple comparisons. These analyses, therefore, reveal that the only site of reliable difference between the younger and older subjects, when comparing the High- and Low-Recency conditions, is that in left-lateral prefrontal cortex, in BA 45. Thus, we feel confident that the activation in this area is the critical substrate underlying the difference in interference resolution between the two age groups.

While older adults do not show as much activation in area 45 as younger adults, they do show evidence of activations in sites that have by now been well-documented as part of a verbal working memory circuit. This is shown in Table 2, which displays the sites of reliable activation in the older adults when subtracting the Minimal-Memory control from the High- and LowRecency conditions. (There were no reliable sites of activation comparing the High- and Low-Recency conditions directly, as our analysis above indicates). Sites such as left-hemisphere posterior parietal and premotor cortex, right-hemisphere cerebellum, and anterior cingulate have regularly been reported in other studies of verbal working memory, and the data in Table 2 agree well with these findings and with our previous report of activations in younger subjects in this same task (Jonides et al., 1998; see also Reuter-Lorenz et al., in preparation for analysis of the effects of age on verbal working memory).

Our results pair a behavioral effect with a site in the brain. Behaviorally, we have shown that older adults are more susceptible to the interfering effect of recent negative probes than are younger adults. Biologically, we have identified this increased interference effect with lower activation in left lateral prefrontal cortex at a site that was prominent in the activations of younger adults in this same task. We conclude that this site is critical to the processes that result in lower interference in younger adults, and, furthermore, that age diminishes the efficacy of this region, resulting in greater susceptibility to the interfering effects of prepotent processes.

One possibility we must consider before ascribing our results to neural differences between young and old is

Table 2. Foci of Activation in the High-Minus Low-Recency, High-Recency Minus Minimal Memory, and Low-Recency Minus Minimal-Memory Comparisons for Older Adults

Stereotaxic coordinates

$\begin{array}{ll}x & z\end{array}$

$z$-score

Brain area
(A) High-Recency minus Low-Recency

\section{no significant activation foci}

(B) High-Overlap minus Minimal-Memory

\begin{tabular}{rrrll}
-28 & -53 & 40 & 6.34 & Left posterior parietal (BA 40) \\
-51 & -4 & 40 & 6.20 & Left premotor (BA 6) \\
37 & -60 & -25 & 5.40 & Right lateral cerebellum \\
3 & -58 & -18 & 5.31 & Right midline cerebellum (vermis) \\
33 & -64 & -47 & 5.07 & Right lateral cerebellum \\
39 & 21 & -2 & 5.04 & Right PFC (BA 47) \\
-17 & -8 & 18 & 4.87 & Left thalamus \\
-37 & -62 & -14 & 4.66 & Left lateral cerebellum \\
-39 & -62 & -25 & 4.64 & Left lateral cerebellum \\
21 & 21 & 18 & 4.40 & Right anterior cingulate (BA 24) \\
$(\mathrm{C})$ Low-Recency minus Minimal-Memory & & & \\
-48 & -4 & 38 & 5.51 & Left premotor (BA 6) \\
-24 & -58 & 43 & 4.73 & Left superior/posterior parietal (BA 7) \\
1 & 17 & 40 & 4.62 & Anterior cingulate (BA 32) \\
24 & 26 & 20 & 4.60 & Right anterior cingulate (BA 24) \\
8 & -71 & -25 & Right midline cerebellum (vermis) \\
\hline
\end{tabular}


that our results are due to differences in hemodynamic responsiveness between the two age groups. D'Esposito, Zarahn, Aguirre, \& Rypma, (in press) have documented that older adults show lower apparent neural activation in sensorimotor cortex in a simple response task that can be traced to higher noise in their hemodynamic responses than younger subjects. This result raises the specter of global differences in blood-flow when comparing age groups that may not be a function of neural activity at all. While this may be so in some cases, we argue that it is not so in ours. First, our analysis procedure normalizes subjects' global blood flow to a common scale, thereby eliminating any overall differences in global flow that may exist between age groups. Second, the activation differences we report in left lateral prefrontal cortex are not a result of differences in variance between groups, but rather a difference in mean activation (using our second ROI analysis, the means of activations in this ROI were $3.53 \%$ in the young and $1.42 \%$ in the old, but the standard deviations of activation were, in fact, a bit lower in the old (2.55\%) than in the young $(3.22 \%)$ ). Third, if differences between age groups were due to global differences in hemodynamic responsiveness, we would have expected to see a difference in many regions, not just the lateral prefrontal region where we found it. Thus, we suspect that although there may be global differences in bloodflow responsiveness as a function of age (whether in mean or variability), we have apparently discovered a locality-specific effect that is not related to these global differences.

We turn, then, to possible cognitive mechanisms that might account for both the greater behavioral effect of recent negative probes on older adults' performance and the activation in left lateral prefrontal cortex for younger but not older adults. In an item-recognition experiment, subjects can base their responses on two indicators. The first is the overall familiarity of a probe (an indirect indication that it is a member of the current target set). The second is a contextual "tag" that marks the item as having been presented in the current list or on the current trial (Monsell, 1978). In the Low-Recency condition, the positive probes have both high familiarity and a current contextual tag, while the negative probes have both low familiarity and a non-current tag, so there is little problem in discriminating positive from negative probes. The same holds true for the positive probes and the non-recent negative probes in the HighRecency condition. It is only the recent negative probes that present a conflict. This conflict arises because the familiarity index is relatively high (because of the recent presentation of the probe in the last trial's target-set and its subsequent rehearsal), while the contextual tag is not current.

In the face of this conflict, there are two hypotheses to account for the behavioral and neurobiological differ- ences between younger and older subjects. By one account, younger subjects may address the conflict between codes by invoking a mechanism that inhibits the less reliable familiarity representation, and this mechanism may be a function of the left lateral prefrontal region that we have identified. The inhibitory process presumably takes time and, therefore, produces the cost in response time that we have documented. As suggested by the lower activation in the left lateral prefrontal region for older adults, these subjects may have a deficit in their inhibitory processing, costing them still extra time in deciding the proper response on the trials with recent negative probes.

An alternative hypothesis relies on the assumption that subjects must pay close attention to the temporal context in which a target item occurred in order to develop an accurate contextual tag for that item. There is ample evidence that lateral prefrontal regions are critical to the proper coding of temporal context, as revealed by deficits in frontal-lobe patients on tasks that interrogate the accuracy of temporal coding (Milner \& Petrides, 1984, Moscovitch \& Winocur, 1995). By this account, the difference between younger and older adults in both behavioral performance and neuroanatomical activation hinges on the idea that older adults suffer a deficit in contextual coding, an idea that is supported in studies showing similarities between advancing age and frontal deficits (Moscovitch \& Winocur, 1995). A decline in accurate coding of the temporal context for a target item will render the decision about whether that item is a member of a recent target-set more difficult. It will render judgments about recent negative probes in the High-Recency condition especially difficult because these are the probes for which contextual information is the only reliable indicator of a correct response. The consequences would be poorer performance on these trials in older than younger subjects, and a concomitant drop in activation in lateral prefrontal cortex.

These two hypotheses present alternative accounts of the role of lateral prefrontal cortex in working memory tasks in which there is interference; sorting these accounts apart will take further research. What is clear from the present study is that younger and older adults differ in behavioral performance and brain activation when resolving interference. Interference resolution is a hallmark of a wide variety of tasks including the Stroop task, go/no-go tasks, and the Anot-B task, and these tasks have shown evidence of involvement of prefrontal cortex (Perret, 1974; Drewe, 1975; Diamond, 1990). Furthermore, tasks that are farther afield have also shown evidence of left lateral frontal involvement when selection among verbal alternatives is required (although the areas involved here may be somewhat different from those involved in tasks that include explicit interference: for example, Desmond, Gabrieli, \& Glover, 1998; Thompson-Schill 
et al., 1998). Unpacking the contributions of various areas of prefrontal cortex in these and other tasks will require detailed analyses of the tasks in question and their underlying processes. We have shown how leverage may be gained on such problems by studying populations, such as older adults, that show compromised skill on these tasks.

\section{METHODS}

\section{Cognitive Task}

Subjects were trained in a practice session that included 180 trials of each of the High- and Low-Recency conditions. Subsequently, they were tested again in a session in which PET measurements were taken. This session included five scans for each of the three conditions (High-Recency, Low-Recency, and Minimal-Memory) presented in a counterbalanced order. In all conditions, the proportions of positive and negative probes were each 50\%. In the High-Recency condition, the negative probes were composed of half recent and half nonrecent negatives, where "non-recent" was defined as a probe that had not been a member of the target set for either of the two previous trials. Also in the HighRecency condition, half the positive probes had been members of the prior trial's target-set and half had not been members of the target set on either of the two previous trials. In the Low-Recency condition, for both positive and negative probes, the probe was never a member of the target set on either of the two previous trials. The same held true for the Minimal-Memory control condition: In this condition, a single letter was presented as the target set, replicated four times in the display to match the visual presentation in the two experimental conditions (see Figure 1). The stimuli (consonants) were presented via Macintosh IIci computer controlled by PsyScope software (Cohen, MacWhinney, Flatt, \& Provost, 1993). Subjects responded by depressing one of two buttons with their right index or middle fingers.

\section{Image Acquisition}

Subjects were tested with a Siemens ECAT EXACT-47 PET scanner with the septa retracted; attenuation was measured, but there was no correction for scattered events. Reconstructing in three dimensions resulted in 47 contiguous slices that were $3.375 \mathrm{~mm}$ apart with resolution of $10 \mathrm{~mm}$ full-width at half-maximum in plane. Head position was verified before each scan. A bolus of $10 \mathrm{mCi}$ of $\left[{ }^{15} \mathrm{O}\right]$ water was delivered intravenously over a 10-sec interval. Acquisition began when the true coincidence rate exceeded half the random coincidence rate. Scans were separated by $8 \mathrm{~min}$ to permit the radiation to return to an acceptable background level.

\section{Image Analysis}

The image-analysis protocol is described in full elsewhere (Jonides, Smith, Marshuetz, \& Koeppe, 1997). It consisted of the following steps: (a) Intra-subject registration to a selected scan was used to correct for motion between scans for each subject (Minoshima et al., 1993a,b); (b) Each subject's images were then normalized to a stereotactic system (Talairach \& Tournoux, 1988; Minoshima, Berger, Lee, \& Mintun, 1992; Minoshima et al., 1993a,b; Minoshima, Koeppe, Frey, \& Kuhl, 1994); (c) A set of subtraction images was then created for each subject to examine the contrasts of interest; (d) The subtraction images were then averaged across subjects; (e) Finally, a pooled estimate of variance was computed by averaging standard deviations for the voxels within the brain, and this was used to calculate a $t$ value for each voxel. The $t$ statistics were evaluated by correcting for multiple non-independent comparisons (Friston, Frith, Liddle, \& Frackowiak, 1991; Worsley, Evans, Marrett, \& Neelin, 1992).

\section{Acknowledgments}

This research was supported in part by a grant from the Office of Naval Research and in part by a grant from the National Institute on Aging, both to the University of Michigan. We thank Leon Gmeindl, Rachael Hippner, Michael Kia, and Elisa Rosier for their assistance with data collection.

\section{Note}

1. One older adult's behavioral data were lost due to equipment malfunction.

\section{REFERENCES}

Carter, C. S., Braver, T. S., Barch, D. M., Botvinick, M. M., Noll, D., \& Cohen, J. D. (1998). Anterior cingulate cortex, error detection, and the online monitoring of performance. Science, 280, 747-749.

Cohen, J. D., MacWhinney, B., Flatt, M. R., \& Provost, J. (1993). PsyScope: a new graphical interactive environment for designing psychology experiments. Behavior Research Methods, Instruments, and Computers, 25, 257-271.

Connelly, S. L., Hasher, L., \& Zacks, R. T. (1991). Age and Reading: The impact of distraction. Psychology and Aging, 6, 533-541.

Cowell, P. E., Turetsky, B. I., Gur, R. C., Grossman, R. I., Shtasel, D. L., \& Gur, R. E. (1994). Sex differences in aging of the human prefrontal and temporal lobes. Journal of Neuroscience, 14, 4748-4755.

Desmond, J. E., Gabrieli, J. D. E., \& Glover, G. H. (1998). Dissociation of frontal and cerebellar activity in a cognitive task: Evidence for a dissociation between selection and search. Neuroimage, 7, 368-376.

D'Esposito, M., Postle, B. R., Jonides, J., Smith, E. E., \& Lease, J. (in press). The neural substrate and temporal dynamics interference effects in working memory as revealed by eventrelated fMRI. Proceedings of the National Academy of Sciences 
D’Esposito, M., Zarahn, E., Aguirre, G. K., \& Rypma, B. (in press). The effect of normal aging on the coupling of neural activity to the bold hemodynamic response. Neurolmage.

Diamond, A. (1990). The development and neural bases of memory functions as indexed by the A non-B and delayed response tasks in human infants and infant monkeys. In A. Diamond (Ed.), The development and neural bases of higher cognitive functions (pp. 267-317). New York: New York Academy of Sciences.

Drewe, E. A. (1975). Go-no go learning after frontal lobe lesions in humans. Cortex, 11, 8-16.

Friston, K. J., Frith, C. D., Liddle, P. F., \& Frackowiak, R. S. J. (1991). Comparing functional (PET) images: The assessment of significant change. Journal of Cerebral Blood Flow and Metabolism, 11, 690-699.

Fuster, J. M. (1989). The prefrontal cortex (3rd ed.). New York: Raven.

Guitton, D., Buchtel, H. A., \& Douglas, R. M. (1985). Frontal lobe lesions in men cause difficulties in suppressing reflexive glances and in generating goal-directed saccades. Experimental Brain Research, 58, 455-472.

Hasher, L., \& Zacks, R. T. (1988). In G. H. Bower (Ed.), The psychology of learning and motivation (pp. 193-224) San Diego, CA: Academic Press.

Haug, H., \& Eggers, R. (1991). Morphometry of the human cortex cerebri and corpus striatum during aging. Neurobiology of Aging, 12, 336-338.

Huttenlocher, P. R. (1979). Synaptic density in human frontal cortex-developmental changes and effects of aging. Brain Research, 164, 195-205.

Jonides, J., Schumacher, E. H., Smith, E. E., Lauber, E. J., Awh, E., Minoshima, S., \& Koeppe, R. A. (1997). Verbal-workingmemory load affects regional brain activation as measured by PET. Journal of Cognitive Neuroscience, 9, 462-475.

Jonides, J., Smith, E. E., Marshuetz, C., \& Koeppe, R. A. (1998). Inhibition in verbal-working memory revealed by brain activation. Proceedings of the National Academy of Sciences, 95, 8410-8413.

Milner, B., \& Petrides, M. (1984). Behavioural effects of frontallobe lesions in man. Trends in Neurosciences, 7, 403-407.

Milner, B., Petrides, M., \& Smith, M. L. (1985). Frontal lobes and the temporal organization of memory. Human Neurobiology, 4, 137-142.

Minoshima, S., Berger, K. L., Lee, K. S., \& Mintun, M. A. (1992). An automated method for rotational correction and centering of three-dimensional functional brain images. Journal of Nuclear Medicine, 33, 1579-1585.

Minoshima, S., Koeppe. R. A., Fessler, J. A., Mintun, M. A., Berger, K. L., Taylor, S. F., \& Kuhl, D. E. (1993a). Integrated and automated data analysis method for neuronal activation studies using O-15 water PET. In K. Uemura, N. A. Lassen, T. Jones, \& I. Kanno (Eds.), Quantification of brain function-tracer kinetics and image analysis in brain PET (pp. 409-418). International Congress Series 1030, Tokyo: Excepta Medica.

Minoshima, S., Koeppe, R. A., Mintun, M. A., Berger, K. L., Taylor, S. F., Frey, K. A., \& Kuhl, D. E. (1993a). Automated detection of the intercommissural line for stereotactic localization of functional brain images. Journal of Nuclear Medicine, 34, 322-329.

Minoshima, S., Koeppe, R. A., Frey, K. A., \& Kuhl, D. E. (1994). Anatomical standardization: Linear scaling and nonlinear warping of functional brain images. Journal of Nuclear Medicine, 35, 1528-1537.

Monsell, S. (1978). Recency, immediate recognition memory, and reaction time. Cognitive Psychology, 10, 465-501.

Moscovitch, M., \& Winocur, G. (1995). Frontal lobes, memory, and aging. Annals of the New York Academy of Sciences, 769, 119-150.

Perret, E. (1974). The left-frontal lobe of man and the suppression of habitual responses in verbal categorical behaviour. Neuropsychologia, 12, 323-330.

Raz, N., Gunning, F. M., Head, D., Dupuis, J. H., McQuain, J., Briggs, S. D., Loken, W. J., Thornton, A. E., \& Acker, J. D. (1997). Selective aging of the human cerebral cortex observed in vivo: Differential vulnerability of the prefrontal gray matter. Cerebral Cortex, 7, 268-282.

Raz, N., Gunning, F. M., Head, D., Dupuis, J. H., \& Acker, J. D. (1998). Neuroanatomical correlates of cognitive aging: Evidence from structural magnetic resonance imaging. Neuropsychology, 12, 95-114.

Raz, N. (in press). Aging of the brain and its impact on cognitive performance: Integration of structural and functional findings. In F. I. M. Craik \& T. A. Salthouse (Eds.), The bandbook of aging and cognition (2nd ed.). Hillsdale, NJ: Erlbaum.

Reuter-Lorenz, P. A., Jonides, J., Smith, E. E., Hartley, A., Miller, A., Marshuetz, C., \& Koeppe, R. (in preparation). Age differences in frontal lateralization of working memory.

Rinne, J. O. (1987). Muscarinic and dopaminergic receptors in the aging human brain. Brain Research, 404, 162-168.

Roberts, R. J., Hager, L. D., \& Hernon, C. (1994). Prefrontal cognitive processes: Working memory and inhibition in the antisaccade task. Journal of Experimental Psychology: General, 123, 374-393.

Salthouse, T. A. (1992). Mechanisms of age-cognition relations in adultbood. Hillsdale, NJ: Erlbaum.

Smith, E. E., Jonides, J., Marshuetz, C., \& Koeppe, R. A. (1998). Components of verbal working memory: Evidence from neuroimaging. Proceedings of the National Academy of Science of the United States of America, 95, 876-882.

Talairach, J., \& Tournoux, P. (1988). Co-planar stereotaxic atlas of the buman brain. Stuttgart: Thieme.

Thompson-Schill, S. L., Swick, D., Farah, M. J., D'Esposito, M., Kan, I. P., \& Knight, R. T. (1998). Verb generation in patients with focal frontal lesions: A neuropsychological test of neuroimaging findings. Proceedings of the National Academy of Sciences of the United States of America, 26, 14792-14797.

Tipper, S. P. (1991). Less attentional selectivity as a result of declining inhibition in older adults. Bulletin of the Psychonomic Society, 29, 45-47.

Warren, L. R., Butler, R. W., Katholi, C. P., \& Halsey, J. H., Jr. (1985). Age differences in cerebral blood flow during rest and during mental activation measurements with and without monetary incentive. Journal of Gerontology, 40, 53-59.

West, R. L. (1996). An application of prefrontal cortex function theory to cognitive aging. Psychology Bulletin, 120(2), 272292.

Worsley, K. J., Evans, A. C., Marrett, S., \& Neelin, P. (1992). A three-dimensional statistical analysis for $\mathrm{rCBF}$ activation studies in human brain. Journal of Cerebral Blood Flow and Metabolism, 12, 900-918. 